\title{
Fast Progression of Diabetic Retinopathy with SARS-CoV-2 Infection
}

\author{
Authors: \\ ${ }^{*}$ Yigit C. Akduman, ${ }^{1}$ William J. Anderson, ${ }^{1}$ Sandeep Saxena ${ }^{2}$ \\ 1. Saint Louis University, St. Louis, Missouri, USA \\ 2. King George's Medical University, Lucknow, India \\ *Correspondence to yiakduman@gmail.com \\ Disclosure: $\quad$ The authors have declared no conflicts of interest. \\ Received: $\quad 09.11 .20$ \\ Accepted: $\quad 15.01 .21$ \\ Keywords: Diabetic retinopathy, macular oedema, neovascularisation, severe acute respiratory \\ syndrome coronavirus-2 (SARS-CoV-2) infection, vitreous haemorrhage. \\ Citation: $\quad$ EMJ Diabet. 2021; DOI/10.33590/emjdiabet/20-00254
}

\begin{abstract}
COVID-19 is caused by severe acute respiratory syndrome coronavirus-2 (SARS-CoV-2) and has been shown to affect a multitude of organ systems. It is often associated with vasculitis or thromboembolic disease with resultant tissue hypoxia. This report presents a case of fast progression diabetic retinopathy in the case of a SARS-CoV-2 infection. The findings conclude that patients with diabetes should be more frequently monitored for emergence or progression of diabetic retinopathy if they present with COVID-19.
\end{abstract}

\section{INTRODUCTION}

COVID-19, which was originally identified in December 2019, has now spread across the globe and was declared a pandemic by the World Health Organization (WHO) in March 2020. COVID-19 is caused by severe acute respiratory syndrome coronavirus-2 (SARS-CoV-2) and has been shown to affect a multitude of organ systems. ${ }^{1}$ It is often associated with vasculitis or thromboembolic disease, and several forms of ocular involvement have also been reported. ${ }^{2}$ Initial studies have shown that the virus can be isolated in eye secretions and may cause ocular surface problems such as conjunctivitis. ${ }^{3,4}$ More recently, retinal involvement has been reported from SARS-CoV-2 infection. Viral RNA of SARS-CoV-2 has been confirmed in post mortem retinal biopsies of infected patients. ${ }^{5}$ Retinal findings on funduscopic examination

are mostly flame-shaped retinal haemorrhages and cotton wool spots. ${ }^{6}$ Additionally, optical coherence tomography (OCT) has shown small hyperreflective lesions at the level of the ganglion cell and inner plexiform layers. ${ }^{7}$ Marinho et al. ${ }^{8}$ suggested that these lesions may be correlated with central nervous system (CNS) findings in SARS-CoV-2 infections as OCT angiography scans were normal in all patients. However, after further investigation and correspondence, the authors discovered that these hyperreflective lesions were found to have an absence of blood flow. ${ }^{9}$ Both of the previously described funduscopic and OCT findings in patients with COVID-19 may be explained by viral invasion and subsequent immune-mediated inflammation. This results in wide spread microvascular ischaemia that affects many organs including the retina. The effect of COVID-19 on the microvasculature could preferentially affect patients with pre-existing microvascular diseases, such as diabetes. This 
case represents an acute, severe progression of diabetic retinopathy in the setting of COVID-19.

\section{CASE REPORT}

This case reports a 49-year-old African-American male who has had poorly controlled Type 2 diabetes mellitus for 10 years, presenting with blurry vision following COVID-19 contraction and hospitalisation. He was being treated with metformin and dulaglutide. His HbA1c was $9 \%$. The patient did not have any history of micro- or macrovascular complications. The primary care physician reported no neuropathy.

During hospitalisation between $16^{\text {th }}$ May and $6^{\text {th }}$ June 2020, the patient's vision in both eyes became blurry. While hospitalised his blood sugar levels were well managed within normal limits. He had moderate COVID-19 symptoms as defined by the U.S. Food and Drug Administration (FDA). ${ }^{10}$ He needed supplemental oxygen with continuous positive airway pressure therapy. He was monitored and did not need a ventilator. He reported being screened in previous years for diabetic retinopathy because he had been diabetic since 2010. He stated no previous eye problems or diabetic retinopathy from his previous eye exams, which were screened by an optometrist. No images were available to compare.
When the patient was first examined in the clinic on $16^{\text {th }}$ June 2020 , his best-corrected visual acuity was 20/30 in the right eye and 20/25 in the left eye. There was no sign of ischaemic optic neuropathy or any specific visual field defect that could have come from optic nerve inflammation. Anterior segment and vitreous examination showed only mild cataracts in each eye and no inflammation. Fundus examination revealed prepapillary and peripapillary subhyaloid vitreous haemorrhages; additionally, it showed intraretinal flame-shaped and dot blot haemorrhages in both eyes. There were hard exudates in the macula in both eyes (Figure 1). The view of most of the optic disc was obscured by the prepapillary haemorrhage in the right eye and there was disc neovascularisation in the left eye. Fluorescein angiography showed late staining of the optic disc mostly blocked by the preretinal haemorrhage in the right eye, and the left fluorescein angiography showed disc neovascularisation (Figure 2). The rest of the retina showed some microaneurysms and moderate peripheral ischaemia in both eyes. The diagnosis was proliferative diabetic retinopathy with diabetic macular oedema in both eyes. OCT showed mild perifoveal focal macular thickening representative of clinically-significant diabetic macular oedema (Figure 3). Other than the thickening of the temporal macula in the OCT, the structural architecture of the retina was preserved.
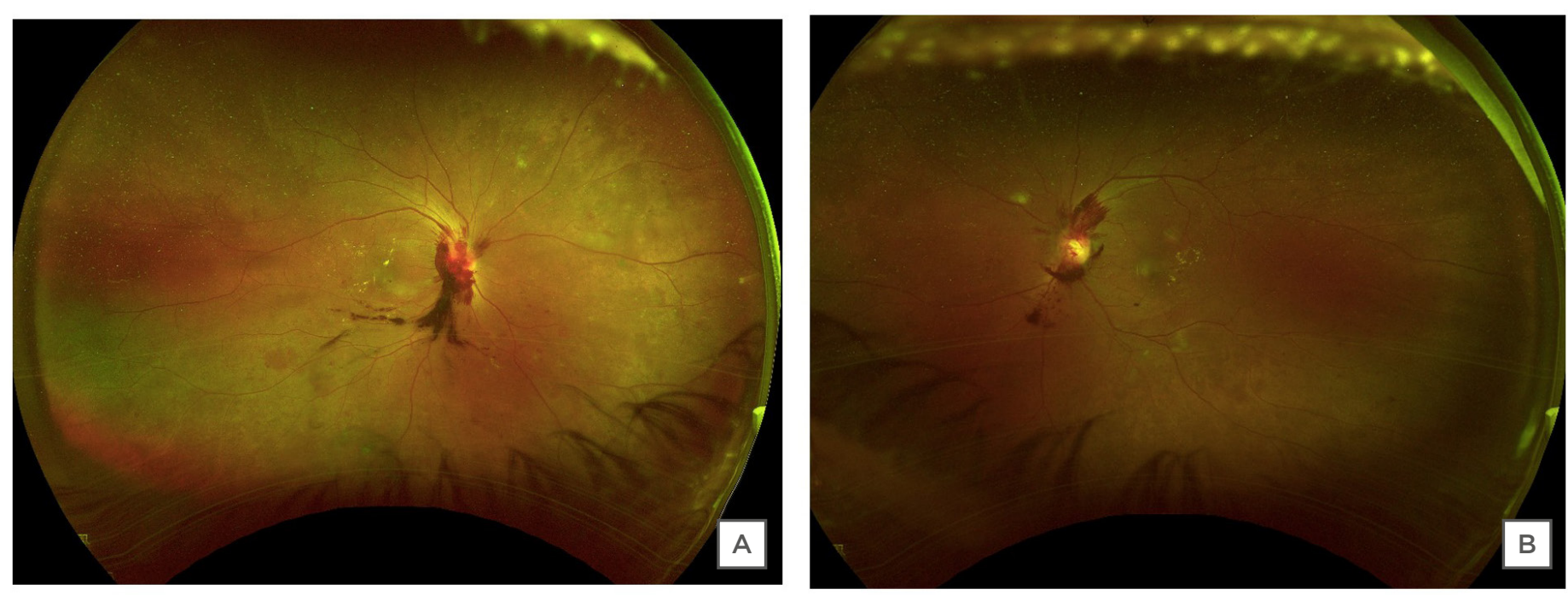

Figure 1: Wide-angle colour fundus photograph of the right $(A)$ and the left $(B)$ eyes.

The images of both eyes reveal preretinal and prepapillary vitreous haemorrhages, flame-shaped, and dot blot retinal haemorrhages, and hard exudates in the macula. 

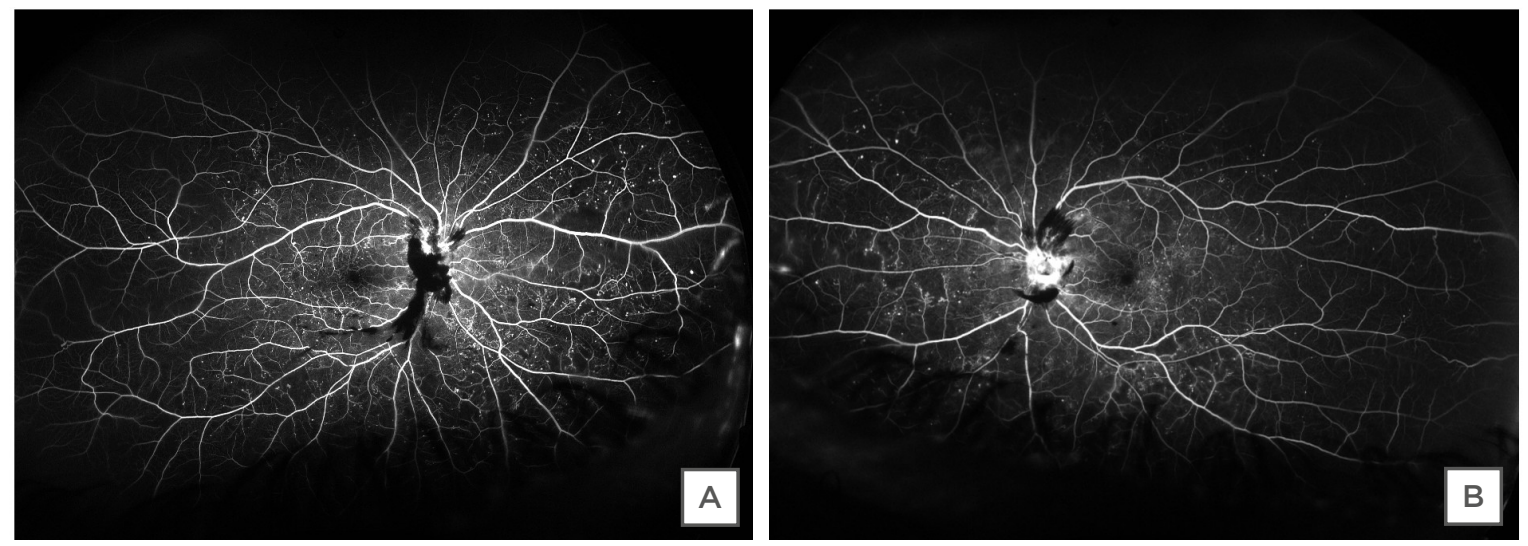

Figure 2: Fluorescein angiography showing the blockage from preretinal haemorrhage, staining of the visible part of the optic disc in the right eye (A), and leaking disc neovascularisation in the left eye (B).

The rest of the retina shows peripheral retinal ischaemia and diffuse microaneurysms.

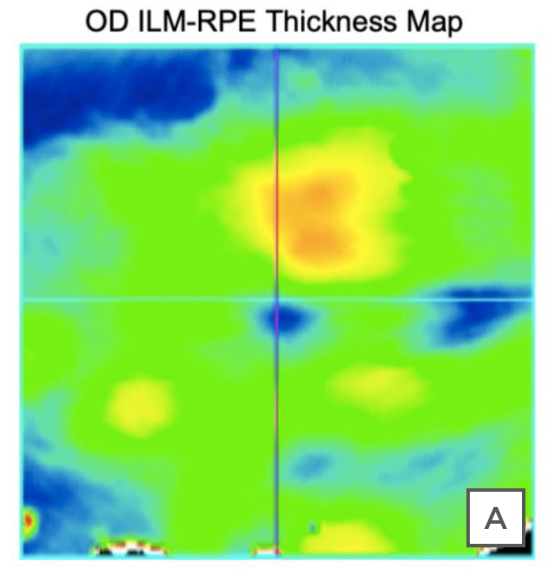

Fovea: 261, 69

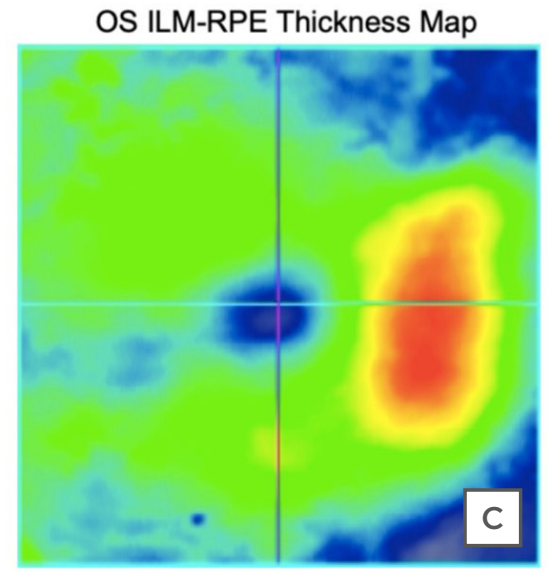

Fovea: 243, 67
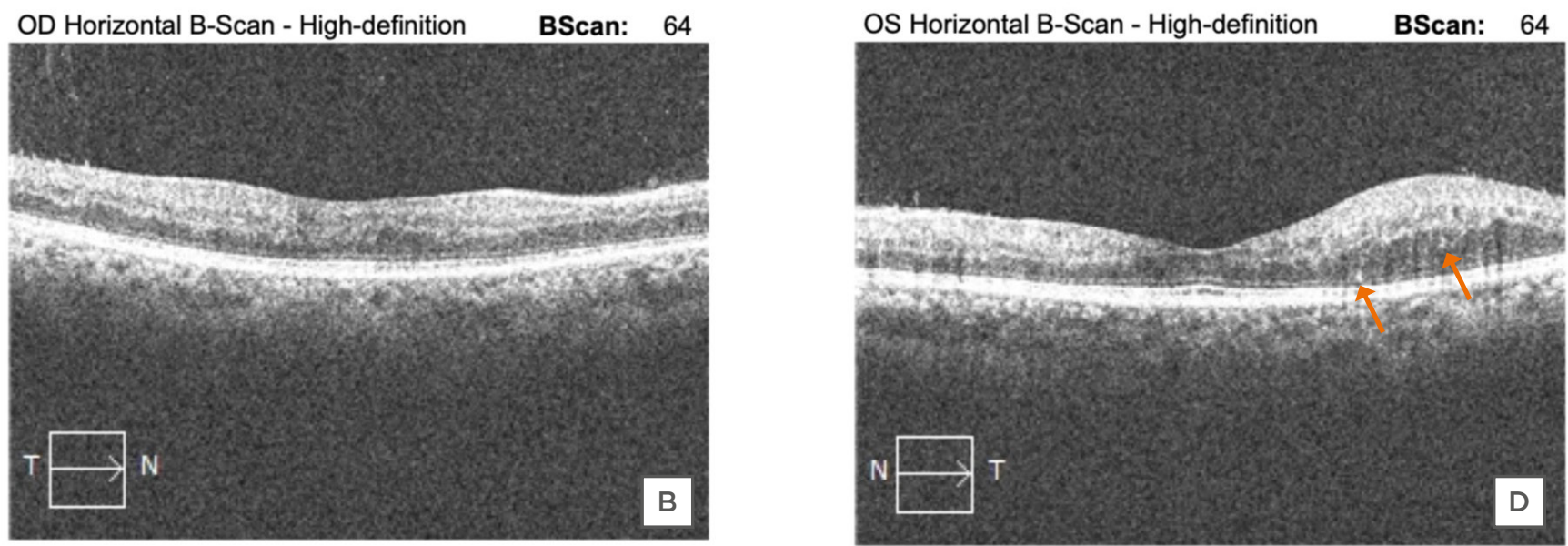

Figure 3: Perifoveal macula is thickened in the right ( $A$ and $B$ ) and left ( $C$ and $D$ ) eyes with fairly well-preserved structural integrity, indicating recent onset macular oedema. Several hyperreflective spots (arrows) are identified as well.

These are similar to those previously described by Pereira et al., ${ }^{6}$ however, hyperreflective spots are non-specific findings that are also commonly seen in diabetic macular oedema alone. They do not necessarily indicate association with COVID-19. 
In the following 3 months, the patient was treated with monthly injections of bevacizumab, focal macular laser in each eye, and peripheral panretinal photocoagulation. This was done for the sake of caution in case the vitreous haemorrhage may have happened from a disc neovascularisation covered by the overlying haemorrhage. On the last examination on $3^{\text {rd }}$ September 2020, his visual acuity was 20/20 in both eyes with near total resolution of macular oedema and partial resolution of the prepapillary haemorrhage.

\section{CONCLUSION}

Previously reported retinal findings in patients with COVID-19 include haemorrhages, cotton wool spots, and hyperreflective ischaemic lesions on OCT. In the largest cohort study of 18 patients, one-half of whom had diabetes, ischaemic retinal findings were present in nearly $50 \%$ of participants. This report describes a patient with diabetes whose retinopathy progressed significantly with COVID-19; the condition progressed from no reported retinopathy or visual symptoms 1 month prior, to high-risk proliferative diabetic retinopathy with vitreous haemorrhage. Existing diabetic vascular compromise may have made his eyes more vulnerable to presumed COVID-19-related vasculitis or venous occlusion. It is thought that this patient's retinopathy may have progressed because of a combined vasculitis and resistance to venous blood flow in the central retinal vein. This may have been because of the SARSCoV-2 infection or the associated secondary effects such as cytokine storm or thrombotic tendency. Similar to previously reported patients, this patient had not received any other known concomitant vasoactive medication that could have further progressed retinal findings. It seems reasonable to suggest that concomitant SARSCoV-2 infection contributed to developing this clinical picture in an eye that had no previously noticeable diabetic retinopathy.

COVID-19 appears to have unique forms of thrombotic and inflammatory processes; in nearly all tissues, thrombosis associated with COVID-19 could be either micro- or macrothrombosis. ${ }^{11}$ This differs from previously described thrombotic and inflammatory processes such as macrophage activation syndrome, disseminated intravascular coagulation, and cytokine release syndrome..$^{12-14}$ Macrophage activation syndrome is rarely associated with thrombosis. Disseminated intravascular coagulation has more disseminated thrombosis associated with low fibrinogen levels. Cytokine release syndrome has different cytokine types and levels than COVID-19related thrombosis and inflammation. In studies done by Ackermann et al. ${ }^{15}$ and Piazza et al.,16 pathologic examination of COVID-19-related thrombotic/necrotic tissue shows evidence of a much higher amount of microvascular injury and microthrombi. ${ }^{15,16}$ Diabetic microvasculature already has compromised circulation and a high tendency for occlusion. This may make diabetic retinopathy more prone to occlusions when microthrombosis and microvascular injury happens as a result of COVID-19. Although this kind of injury has been reported in other tissues, it has not yet been directly observed pathologically in cases of diabetic retinopathy. In this report, some diabetic microvasculature may have already been compromised and easily occluded when the patient contracted COVID-19. Similar specific accelerated thrombotic processes have been recently shown to be particularly apparent in the lungs and CNS." The retina is an extension of the CNS and therefore, the retinal and optic nerve vasculature may similarly be particularly susceptible to such a thrombotic event. The new nomenclature suggested by Bilgin et al., ${ }^{17}$ 'inflammatory thrombosis with immune endotheliitis-ITIE', may also apply to the case presented here as the contributory factor for the progression of diabetic retinopathy with SARSCoV-2 infection. This case and the previously reported cases of retinal findings secondary to retinal ischaemia, even in the absence of diabetic retinopathy in patients with COVID-19, warns us that diabetic retinopathy may progress faster than expected in patients infected with SARS-CoV-2.6.7 Patients with diabetes should be more frequently monitored if they get COVID-19 for emergence or progression of diabetic retinopathy. 


\section{References}

1. Lai CC et al. Extra-respiratory manifestations of COVID-19. Int J Antimicrob Agents. 2020;56(2):106024.

2. Klok FA et al. Incidence of thrombotic complications in critically ill ICU patients with COVID-19. Thromb Res. 2020;191:145-7.

3. Mahmoud $\mathrm{H}$ et al. Assessment of coronavirus in the conjunctival tears and secretions in patients with SARS-CoV-2 infection in Sohag Province, Egypt. Clin Ophthalmol. 2020;14:2701-8.

4. Wu P et al. Characteristics of ocular findings of patients with coronavirus disease 2019 (COVID-19) in Hubei Province, China. JAMA Ophthalmol. 2020;138(5):575-8

5. Casagrande $\mathrm{M}$ et al. Detection of SARS-CoV-2 in human retinal biopsies of deceased COVID-19 patients. Ocul Immunol Inflamm. 2020;28(5):721-5.

6. Pereira LA et al. Retinal findings in hospitalised patients with severe COVID-19. Br J Ophthalmol. 2020;DOI:10.1136/ bjophthalmol-2020-317576.

7. Marinho PM et al. Retinal findings in patients with COVID-19. Lancet. 2020;395(10237):1610.

8. Chahbi $M$ et al. Mechanisms of retinal damage in patients with COVID-19. J Fr Ophtalmol. 2020;43(10):e355-6.

9. Marinho PM et al. Seeking clarity on retinal findings in patients with COVID-19 - authors' reply. Lancet. 2020;396(10254):e40.

10. Polack FP et al. Safety and efficacy of the BNT162b2 mRNA Covid-19 vaccine. N Engl J Med. 2020;383(27):2603-15.

11. Minoia F, Ravelli A. Evaluating diagnostic criteria for macrophage activation syndrome in patients with adult onset Still's disease. Response to: 'Comparison of MS score and HScore for the diagnosis of adult-onset Still's disease associated macrophage activation syndrome' by Zhang et al. Ann Rheum Dis. 2020;DOI:10.1136/ annrheumdis-2020-218033.

12. McGonagle D et al. Immune mechanisms of pulmonary intravascular coagulopathy in COVID-19 pneumonia. Lancet Rheumatol. 2020;2(7):e437-45.

13. Webb BJ et al. Clinical criteria for COVID-19-associated hyperinflammatory syndrome: a cohort study. Lancet Rheumatol. 2020;2(12):e754-63

14. Ackermann $\mathrm{M}$ et al. Pulmonary vascular endothelialitis, thrombosis, and angiogenesis in Covid-19. N Eng Med. 2020;383(2):120-8.

15. Piazza G, Morrow DA. Diagnosis management, and pathophysiology of arterial and venous thrombosis in COVID-19. JAMA. 2020;324(24):2548 9.

16. Vasquez-Bonilla WO et al. A review of the main histopathological findings in coronavirus disease 2019. Hum Pathol. 2020:105:74-83.

17. Bilgin E, Ertenli Al. Proposal of a new nomenclature for the underlying pathogenetic mechanism of severe coronavirus disease-19: "Inflammatory Thrombosis with Immune EndotheliitisITIE". Rheumatol Int. 2021;DOI:10.1007/ s00296-020-04768-1. 